Fabrication of periodic nanostructures by single-point diamond turning with focused ion beam built tool tips

This article has been downloaded from IOPscience. Please scroll down to see the full text article.

2012 J. Micromech. Microeng. 22115014

(http://iopscience.iop.org/0960-1317/22/11/115014)

View the table of contents for this issue, or go to the journal homepage for more

Download details:

IP Address: 161.112.232.219

The article was downloaded on 28/09/2012 at 09:40

Please note that terms and conditions apply. 


\title{
Fabrication of periodic nanostructures by single-point diamond turning with focused ion beam built tool tips
}

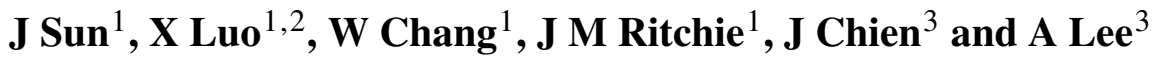 \\ ${ }^{1}$ School of Engineering and Physical Sciences, Heriot-Watt University, Edinburgh, EH14 4AS, UK \\ ${ }^{2}$ School of Computing and Engineering, University of Huddersfield, Huddersfield, HD1 3DH, UK \\ ${ }^{3}$ Metal Industries Research and Development Centre, Kaohsiung, 81160, Taiwan \\ E-mail: x.luo@hud.ac.uk
}

Received 23 April 2012, in final form 7 August 2012

Published 26 September 2012

Online at stacks.iop.org/JMM/22/115014

\begin{abstract}
Periodic nanostructures have been widely used on emerging nano-products such as plasmonic solar cell and nano-optics. However, lack of cost-effective fabrication techniques has become the bottleneck for commercialization of these nano-products. In this work, we develop a scale up approach to fabricate high-precision nanostructures in large area. In this method, a nano-scale single crystal diamond (SCD) tool is produced by focused ion beam (FIB) machining. The nano SCD tool is then further applied to cut periodic nanostructures using single-point diamond turning (SPDT). A divergence compensation method and surface topography generation model forms a deterministic FIB fabrication approach. It has been used to generate four periods of the required periodic nano-grating structures (with a minimal dimension of $150 \mathrm{~nm}$ ) on a normal SCD tool tip and achieves $10 \mathrm{~nm}$ form accuracy. The contribution of the beam tail effect has also been evaluated by using the surface topography simulation method. The fabricated diamond tool is then applied to obtain nano-grating on an electroless nickel substrate in a total area of $5 \times 2 \mathrm{~mm}^{2}$ through SPDT. The whole SPDT machine process only takes $2 \mathrm{~min}$ (with a material removal rate up to $1.8 \times 10^{4} \mu \mathrm{m}^{3} \mathrm{~s}^{-1}$ ). Due to the elastic recovery that occurred upon the workpiece material, the practical cutting width is $13 \mathrm{~nm}$ smaller than the tool tip. The machining trial shows it is very promising to apply this scale up nanofabrication approach for commercialization of nano-products which possess period nanostructures.
\end{abstract}

(Some figures may appear in colour only in the online journal)

\section{Introduction}

Nowadays, nanotechnology is undoubtedly at the frontier of both scientific and engineering research. Great endeavours on the design and fabrication of nanostructures on functional materials are being made for innovative applications. Periodic nano-gratings and nano-dots are typical nanostructures for the excitation of surface plasmon resonance (Atwater and Polman 2010, Liu et al 2005), which is the basis of many resonancecoupling based plasmonic devices, such as the plasmonic solar cell (Akimov and Koh 2010, Mokkapati et al 2009) and the surface-enhanced Raman spectroscopy (Clark and Cooper 2011), for massively enhancing the adsorption of the signal onto planar metal surfaces. For ubiquitous application of these innovative devices, nanostructures have to be manufactured in a repeatable and cost-effective manner while maintaining a high precision.

Several micro- and nano-fabrication techniques are currently used to obtain micro- and nanostructures: FIB machining is an ideal technique for obtaining 3D structures at micro- and nano-scale, but it is limited by the low throughput. Scanning probe lithography is a machining approach in which a stylus is moved mechanically across a substrate surface to form a pattern, but this method is limited by low throughput and only materials that are softer than the stylus can be machined (Yan et al 2010). Optical lithography 


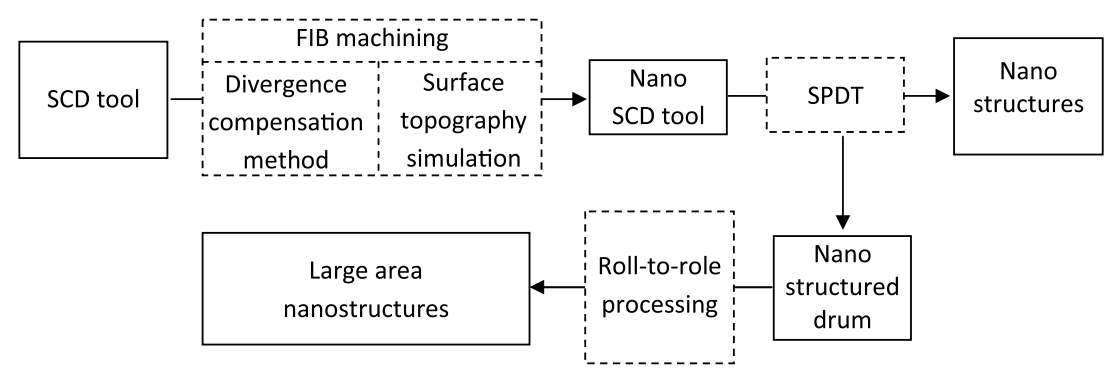

Figure 1. Block diagram of the proposed nanofabrication process.

and electron beam lithography are enabling technologies to fabricate structures over a larger area than FIB machining; however, they are only suitable for the fabrication of $2 \mathrm{D}$ or $2 \frac{1}{2} \mathrm{D}$ structures on a planar surface. Laser machining is another technique used for micro- and nano-fabrication, but the attainable machined surface roughness is much higher than the above fabrication techniques (Dubey and Yadava 2008). On the other hand, single-point diamond turning (SPDT) has been exploited successfully to generate a wide range of high-precision micro-optical structures on discrete optical components (Cheung and Lee 2003). The crystalline structure of diamond permits the fabrication of cutting tools with very sharp cutting edges which result in the nanometre level surface finish for these microstructures after a single cutting pass. However, current attainable size of the diamond cutting tools by routine machining techniques limits the application of SPDT in nanofabrication, although there are a few successful cases in the fabrication of micro-optical components (Xu et al 2010). Nanoimprint lithography is another method that suits for mass production (Chou et al 1996). It is capable of producing sub $10 \mathrm{~nm}$ features (Chou et al 1997) over large area. But these features can only be transferred to proper polymer resist. Other advanced manufacturing techniques such as reactive ion etching (RIE) and micro-contact printing $(\mu \mathrm{CP})$ are generally used to further develop the features onto other materials (Chou et al 1996, Chou et al 1995). But during the transferring process, both the surface roughness and form accuracy will be degraded. Lack of cost-effective fabrication approach to generate high-precision nanostructures in large area has become the bottleneck for commercialization of these innovative nano-products.

In this work, we present a scale up approach to costeffectively generate high-precision nanostructures in large area. For the first time, we demonstrate a FIB built nano SCD tools with a tip width of $150 \mathrm{~nm}$, while a $10 \mathrm{~nm}$ form accuracy is achieved. This approach can be illustrated in figure 1. A normal SCD tool used in SPDT will be used as a tool blank. Fabrication of one to several periods of the required periodic nanostructures on the SCD tool tip is the most critical part of this approach. A deterministic FIB fabrication method is developed to achieve the required accuracy of the nanoscale SCD tool. Periodic nanostructures are then replicated on (planar or curved) the substrate by applying the developed nanoscale SCD tools through SPDT. More important, this approach can also be used to fabricate nanostructures on a precision drum, which can be further applied to imprint nanostructures over extreme large area by a roll-to-roll process (Krebs et al 2009).

\section{Challenges in the fabrication of nanoscale SCD tools}

In the last few years, the literatures report that novel fabrication techniques such as laser (Nakamura and Nakai 1993) and focused ion beam (FIB) methods (Picard et al 2003, Ding et al 2008, Xu et al 2010, Evans et al 2006, Allen et al 2009) can be used to shape diamond. FIB machining is an ideal technology for obtaining micro- and nanostructures (Volkert and Minor 2007, Tseng 2005). The feasibility of fabricating micro-tools by FIB sputtering has been successfully demonstrated by many researchers. Micro-cutting and micro-milling tools made of diamond (Ding et al 2008), cobalt M42 high-speed steel and C2 tungsten carbide (Adams et al 2000, Adams et al 2001) were successfully developed in the past. The key dimensions of these tools ranged from 15 to $100 \mu \mathrm{m}$. Varieties of tool geometries, such as single-tipped single crystal diamond (SCD) tool (Ding et al 2008), dual-tipped SCD tool (Picard et al 2003), diffractive-optical-elements shaped micro-tool and hemispherical micro-tool (Xu et al 2010) have been produced with the help of FIB machining. However, the production of SCD cutting tools with nanometre level features by FIB is still a difficult task.

There is no doubt that the form accuracy of the fabricated diamond tool is essential to its cutting performance during the manufacturing of nanostructures. However, the achievable form accuracy of the nanoscale SCD tools can be significantly reduced particularly where the size of the structures is at the same level as the beam spot size. Some nano-effects that are generally negligible in the micro-fabrication process have to be taken into consideration. In the fabrication of nanoscale SCD tools, there are five main issues which need to be addressed.

\subsection{Ion beam drifting during the fabrication process}

Since diamond is a type of dielectric material, an additional electric field will be generated when it is bombarded by charged particles such as gallium ions. Consequently, the incident ion beam will be deflected before it reaches the diamond surface, which greatly deteriorates the machining accuracy. Therefore, the accumulated charges on diamond must be conducted out of the system efficiently. 


\subsection{Formation of a sharp cutting edge}

According to the machining mechanics of SPDT process, the cutting edge needs to be extremely sharp to improve the machined surface finish. Additionally, a blunt cutting edge can also cause more tension applied on the tool tip and lead to reduction of the tool life. However, edges of the face formed by the ion beam are always rounded due to the Gaussian distribution of the beam. To make a nano-SCD tool that has super cutting performance, the fabrication of faces with sharp edges becomes the prime task. A sharp cutting edge can be formed by adjusting the orientation of the tool face relative to the incident ion beam. It has been reported that the cutting edge near to incident ion beam is rounded off due to the truncation of Gaussian beam profile, while the other edge away from the beam becomes much sharper (Vasile et al 1996). Paying careful attention is required in order to produce a sharp cutting edge when using FIB sputtering, especially for SCD tools with complex structures or multiple cutting edges, the orientation of the tool face has to be adjusted several times. For SCD tools with single tip and double tips, the orientation of the tools has to be adjusted four (Ding et al 2008) and six (Picard et al 2003) times, respectively. In general, the tool rotation/sputter sequence and the location of faces during the FIB fabrication process have profound influences on the tool cutting performance.

\subsection{Ripples generated on the diamond surface}

Ion-bombardment of solid surfaces is known to cause the formation of periodically-modulated structures often referred to as ripples (Bradley and Harper 1988). The ripples on the diamond tool surface will increase its surface roughness and, therefore, must be eliminated.

\subsection{Redeposition effect in the FIB sputtering process}

The redeposition effect is an inevitable effect which occurs during the FIB sputtering process. Some of the sputtered atoms will attach to the sidewall and bottom of the machined structures to result in the divergence to the intended shape. Surface finish of diamond tools is also degraded. The redeposition ratio $^{4}$ mainly depends upon the aspect ratio, the profile of the fabricated structures and the fabrication parameters. In a large proportion of FIB milling applications, the redeposition effect should be suppressed as much as possible.

\subsection{Contribution from the beam tail effect}

The beam tail effect is generated from the Gaussian distribution of the ion beam. The contribution depends on the beam spot size and the pitch between adjacent scanning pixels. Extra material removed by the dose contributed by the beam tail will degrade the form accuracy of nano-SCD tools.

${ }^{4}$ Redeposition ratio is defined as the ratio between the number of redeposited atoms and the number of total sputtered atoms.

\section{Experimental details}

The fabrication of nano-SCD was carried out on a dualbeam FIB system (FEI Quanta 3D FEG). In this work, the system was operated at a fixed acceleration voltage of $30 \mathrm{kV}$. A SCD tool with a tip width of $5.89 \mu \mathrm{m}$ was prepared for the nanofabrication. The tool blank was mounted on a five-axis motorized stage having a $300 \mathrm{~nm}$ motion resolution and $360^{\circ}$ rotational motions with a minimum of $0.1^{\circ}$ increments. The secondary electrons emitted during the FIB fabrication process were collected to provide images of the fabricated tool for the purpose of process monitoring. Periodical chisel structures with a height of $0.6 \mu \mathrm{m}$ and a tip width of $0.15 \mu \mathrm{m}$ are designed on the tool tip. In order to guarantee the expected performance and the capability required in the desired products, the deviation tolerances of these nanostructures need to be confined within $\pm 5 \%$. The experimental process consisted of four steps, including sample preparation, rough cutting process, cleaning process and the final cutting process. Schematic procedures to form a nano-SCD tool from an original diamond tip using the FIB sputtering is shown in figure 2 .

Before the FIB machining process, the diamond tool needs to be carefully prepared to suppress the image drift. The bottom part of the diamond was painted with a layer of silver (Quick drying silver paint @ Agar Scientific). The painted part directly contacted with an aluminium stage over a broad contact area in order to increase the charge conducting efficiency. A layer of platinum with a thickness of $3 \mathrm{~nm}$ was deposited onto the tip of the diamond tool using FIB induced deposition. The advantage of this method is that the charged particles can be conducted out of the SCD tool efficiently. Meanwhile, since the platinum was only coated on the flank face of the SCD tool, the rake face was kept clear and, therefore, fewer impurities would be transferred to the work piece cut by this SCD tool.

During the fabrication process, a beam of ions with a current of $50 \mathrm{pA}$ scanned over the diamond tip to remove the redundant material. To avoid the over sputtering effect and to minimize the erosion caused by beam tail on the cutting edge, the ion dose at each point on the clearance face needs to be precisely controlled proportionally to the local depth of the diamond block. Therefore, a three-dimensional FIB fabrication process was carried out. In this work, a divergence compensation method (Sun et al 2011b) was applied to optimize the FIB machining parameters. Since the minimal dimension on the diamond tip is only $150 \mathrm{~nm}$, the beam diameter must be kept much less than this value in order to guarantee machining accuracy. In this case, the beam diameter was $19 \mathrm{~nm}$ which was approximately $1 / 8$ of the minimal dimension on the tip. The pixel spacing was matched to the beam diameter so that the beam overlap effect could be suppressed (Sun et al 2011b). In order to minimize the contribution from the beam tail, a positive offset along the normal direction of the tool's profile is necessary. The offset distance was set at $25 \mathrm{~nm}$ based on the surface topography simulation results.

The rough cutting process was followed by ion beam cleaning process to finely polish the profile of the chisel 

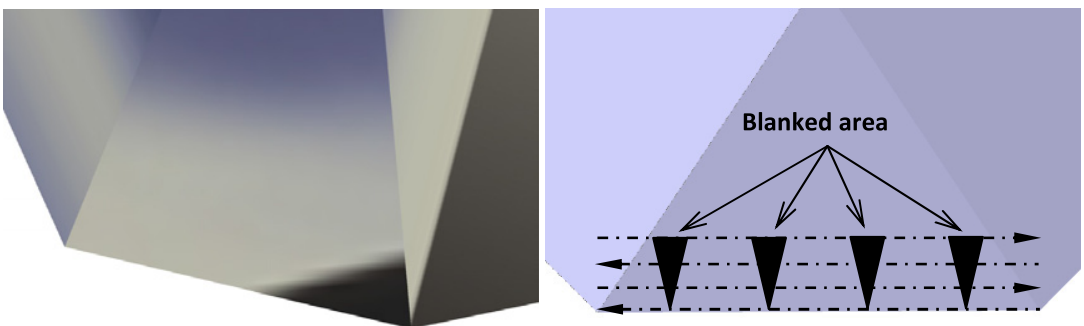

(a)
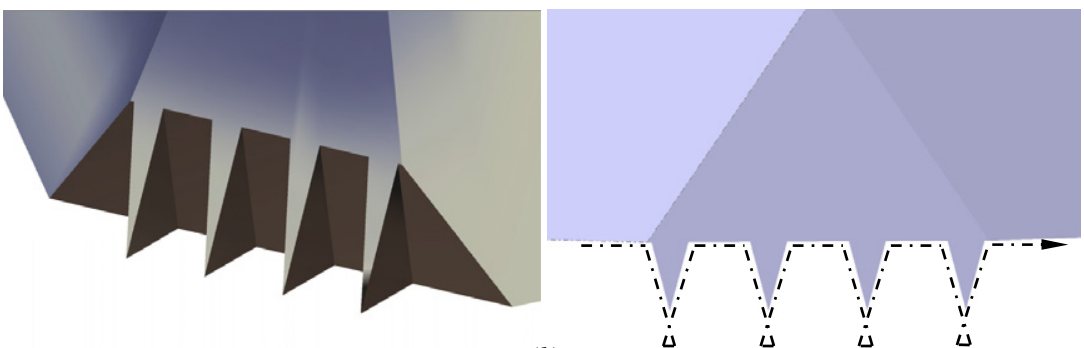

(b)
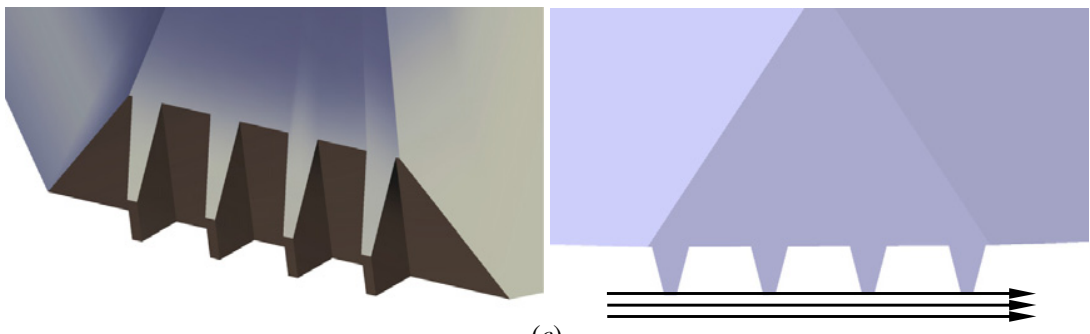

(c)

Figure 2. The FIB machining procedure for nano-SCD tools with periodical chisel structures: $(a)$ fabrication of trapezoidal structures on the diamond tip; $(b)$ the ion beam cleaning process and $(c)$ cutting off the vertexes of the triangular tips.

structures The beam scanning path followed the edge of the designed profile to minimize the redeposition effect. For each cutting line, the beam repeatedly scanned over with dwell times proportional to the local thickness of the diamond tip. Vertexes of the triangular diamond tips were cut off by applying a low ion current and the periodical chisel structures with a height of $600 \mathrm{~nm}$ were obtained.

\section{Results and discussion}

\subsection{Ripples on the surface}

Periodical ripples can be observed on a diamond surface when it is bombarded by high-energy ions. The underlying mechanism of this ripple formation can be traced to a surface instability caused by the competition between curvaturedependent sputtering and surface diffusion processes. The ripples are generally produced by off-normal incident ion beam. The orientation of the ripple depends on the beam incident angles (Bradley and Harper 1988). For incident angles less than a critical angle from the normal, the wave vector of the modulations is parallel to the component of the ion beam in the surface plane. The wave vector is perpendicular to this component for the incident angles close to grazing. The wavelength of these ripples typically depends on the ion incident angle. According to Bradley and Harper's theory (Bradley and Harper 1988), the wavelength approaches infinity when the ion incident angle approaches $90^{\circ}$. That is to say that ideally a ripple-free surface can be formed when the ion incident angle is $90^{\circ}$.

To investigate the ripples formed on diamond surface, a diamond block was sputtered by a beam of $30 \mathrm{keV}$ incident ions. The incident angles varied from $0^{\circ}$ to $90^{\circ}$. For each incident angle, $2.8 \times 10^{11}$ ions were incident on the diamond substrate with different ion currents. The surface topographies of the diamond substrate were measured by an atomic force microscope (AFM) and were shown in figure 3. Experimental results reveal that for certain ion dose, the wavelength of the ripples increases with the ion incident angle when it is less than the critical angle from the normal. As shown in figures 3(a)-(c) when $30 \mathrm{pA}$ gallium ions are incident on the diamond substrate with incident angles of $10^{\circ}, 30^{\circ}$ and $50^{\circ}$, the wavelength of the ripples keeps increasing from 45 to $140 \mathrm{~nm}$. This is because the flux of the incident ions decreases with the increase of the incident angle. According to Bradley and Harper's theory, the wavelength of the ripples is inverse proportional to the ion flux. The wavelength therefore increases with the ion incident angle, which is consistent with the prediction of Bradley and Harper's theory. Further investigation also shows that the magnitude of the ripples increases with the incident ion current. Figures $3(d)-(f)$ show the surface topographies of the ripples generated under $85^{\circ}$ incidence with different ion currents of $30 \mathrm{pA}, 100 \mathrm{pA}$ and $1 \mathrm{nA}$. In this case, the wave vectors of the ripples are perpendicular to the component of the ion beam in the surface plane. The measurement results show that the magnitudes of these ripples are 5, 80 and 


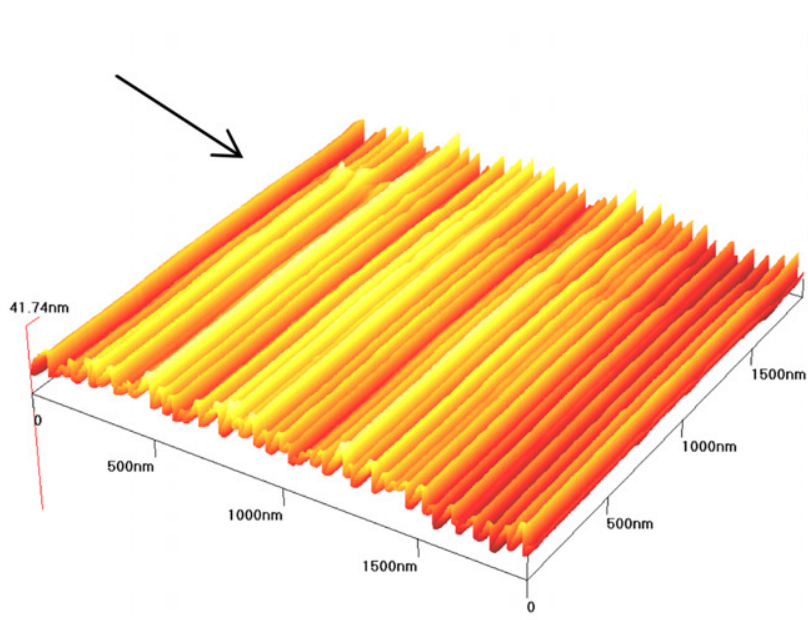

(a)

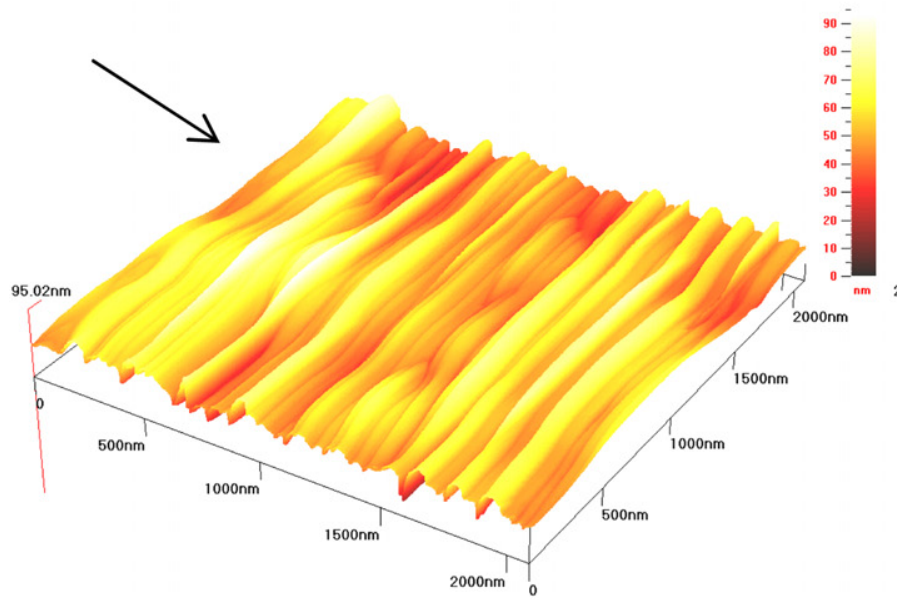

(c)

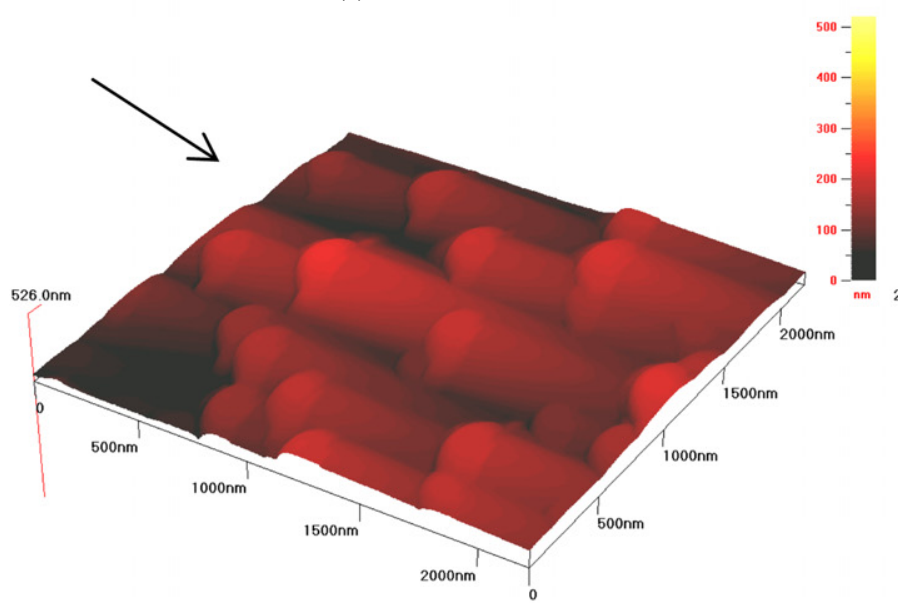

(e)

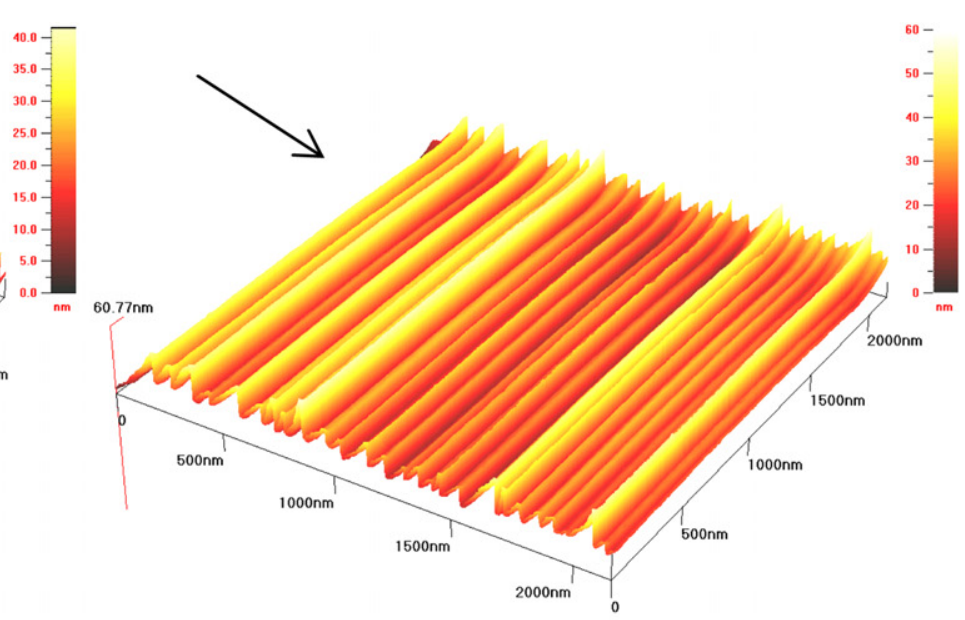

(b)

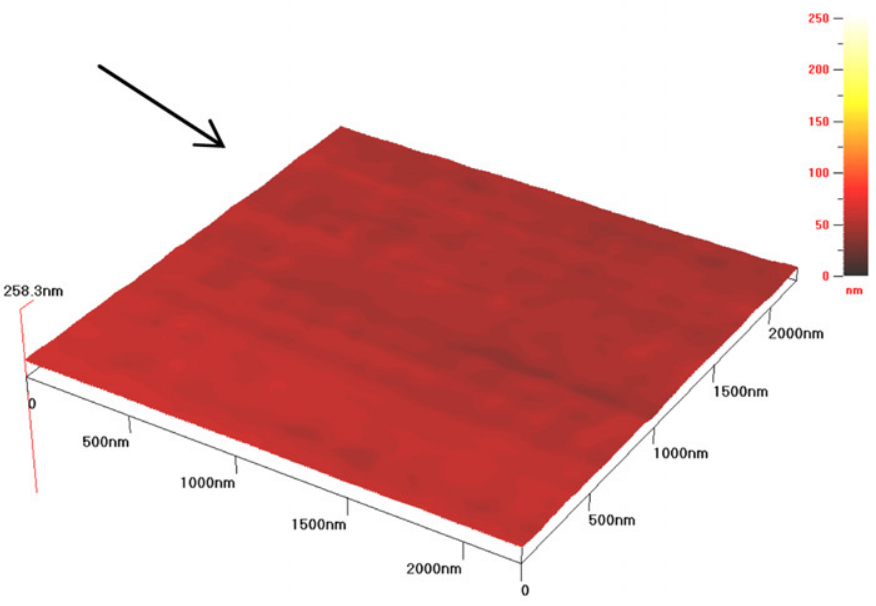

(d)

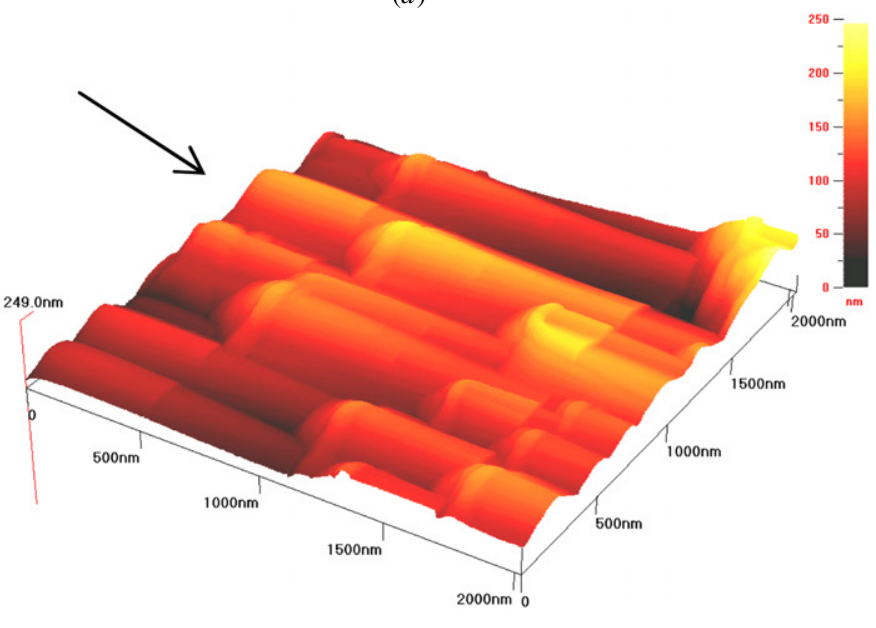

(f)

Figure 3. Surface topographies of diamond sputtered by $30 \mathrm{keV}$ gallium ions under different incident angles $(\theta)$ and ion currents $(I)$ : $(a) I=$ $30 \mathrm{pA}, \theta=10^{\circ} ;(b) I=30 \mathrm{pA}, \theta=30^{\circ} ;(c) I=30 \mathrm{pA}, \theta=50^{\circ} ;(d) I=30 \mathrm{pA}, \theta=85^{\circ} ;(e) I=100 \mathrm{pA}, \theta=85^{\circ}$ and $(f) I=1 \mathrm{nA}, \theta=85^{\circ}$. The arrows indicate the beam direction.

$100 \mathrm{~nm}$, respectively. This phenomenon can be qualitatively explained by the following reason. As the ion beam strikes on an inclined surface, the energy deposited at the local sputtering point primarily comes from the adjacent sputtering points. The uneven energy deposition on the surface induces the formation of the surface ripples. Under high ion current bombardment, the energy deposition rate is much higher. Therefore, the magnitude of the ripples in each scanning pass is higher. As the ion current decrease to certain threshold, the surface ripples generated in each scanning pass can be counteracted by the redeposition effect and the ions reflection along the surface. The magnitude of the ripples is therefore minimized under such circumstance. As it is shown in figure $3(d)$, the surface ripples are almost eliminated under a low current bombardment. A 


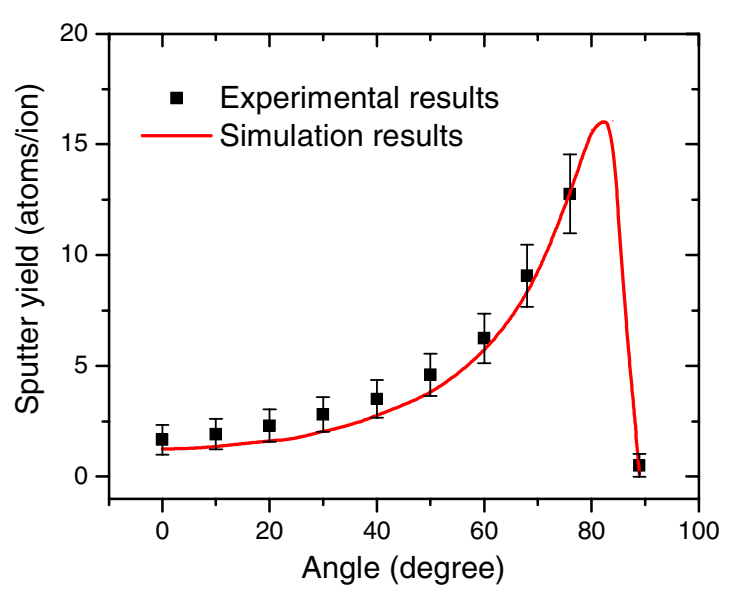

Figure 4. Plot of the sputter yield of diamond (atoms/ion) versus ion beam incidence angle.

further increase of the ion incident angle from $85^{\circ}$ towards $90^{\circ}$ results in a ripple free surface. This result reveals that grazing incidence with low ion current is helpful in forming a ripple free diamond surface.

\subsection{Evaluation of sputter yield}

The machining efficiency in the FIB nanofabrication process depends on the sputter yield. The diamond sputter yield under various incident angles needs to be determined. In this work, the sputter yield was firstly calculated using a Monte Carlo simulation code-TRIDYN (Möller and Eckstein 1984, Möller et al 1988) and then compared with values obtained from experiment. The calculations modelled impingement of $30 \mathrm{keV}$ gallium ions onto a flat diamond surface. The heat of sublimation, namely $7.41 \mathrm{eV}$ was taken as the surface binding energy of diamond. The bulk binding energy and displacement energy were assumed to be 3 and $28 \mathrm{eV}$ (Ziegler). In the experiment, a series of microcavities were fabricated on a flat diamond substrate with different incident angles ranging from $0^{\circ}$ to $90^{\circ}$. The ion current and sputtered cavity volume were measured by a KEITHLEY 6485 picoammeter and an AFM (HYSITRON TI900 Tribolndenter), respectively. As it is shown in figure 4, the variation of the calculated sputter yield agrees with the experimental results, but with a systematic divergence. This divergence is primarily attributed to the ripples generated during the sputtering process. Due to the existence of the ripples with wavelengths comparable with the beam diameter, the ion incident angle varies with the surface profile. For certain incident angles, the measurement results are actually an integration of the sputter yield under all the possible angles all over the bottom surface and sidewalls. However, this dynamic change on the surface profile is not taken into consideration in the calculation.

\subsection{The redeposition effect}

For concave structures with high aspect ratio, the sputtered atoms are less likely to escape from the cavity and are therefore redeposited onto the sidewalls. The redeposition effect can be ignored if there are only non-concave structures on the tool tip. However, for tools with a notch or a jagged shape in the cutting edge, small amounts of material from one side of the edge could redeposit at the opposite side. As shown in figure 5, a rectangular cavity with a length of $750 \mathrm{~nm}$ is fabricated on the edge of a diamond block. In order to minimize the ripples on the sidewalls and improve the machining accuracy, a polishing process with an ion current of $30 \mathrm{pA}$ was carried out. The polishing process followed three independent steps as shown in figure 5( $a$ ) and is called as an 'edge to edge' process, i.e. one edge is finely polished followed by another. An SEM image of the three polished edges is shown in figure $5(b)$, where the bulk of amorphous material is found on the first polished edge due to the redeposition effect. The machining accuracy is therefore degraded. Special scanning strategies need to be developed to minimize the redeposition effect when polishing nanoscale SCD tools. A 'multi-edge' polishing method can be applied in an FIB cleaning process. Multi-edge polishing means the edges that need to be polished are processed simultaneously. As illustrated in figure $5(c)$, the beam spot is controlled during scanning along the overall profile of the rectangular structure. The scanning path started from a position $100 \mathrm{~nm}$ away from the edge. Focused ions are deflected along the scanning path until a specific milling depth is achieved. The ion beams them move onto the next scanning path. The pitch between the adjacent scanning paths is $10 \mathrm{~nm}$. The scanning path approaches the edges and stops until the scanning path overlapped with the edges. The advantage of this method is that the deposited atoms on each edge are immediately removed by the following scan. Therefore, the redeposition effect is suppressed. The structure polished by this method is shown in figure $5(d)$; comparing with figure $5(b)$, the polished structure possesses much better form accuracy.

\subsection{Evaluation of the beam tail effect}

Due to the scattering of the ions emitted from the liquid metal ion source and the limitation in the focusing capability of the ion column, the ion beam has a finite size and is usually considered as a Gaussian distribution with a circular cross section. The beam diameter is defined as the fullwidth at half-maximum (FWHM) of the Gaussian distribution. However, the diameter of a beam profile does not provide a sufficient description of the beam characteristic. The actual cutting line width is generally bigger than the beam diameter. It is necessary and useful to develop sensitive method for evaluating the effect caused by beam tail in the FIB machining process. In this work, the contribution from beam tail is evaluated by surface topography simulation based on a level set method (Sun et al 2011a). The level set method (Sethian 2000) is a numerical technique for tracking interfaces and shapes. The advantages of level set method are that one can perform numerical computations involving curves and surfaces on a fixed Cartesian grid without having to parameterize these objects, and the topological merging and breaking, sharp gradients and cusps can form naturally. All these advantages make level set method a more great and robust tool than other conventional simulation methods for modelling time-varying objects. The central mathematical idea of level set method is 

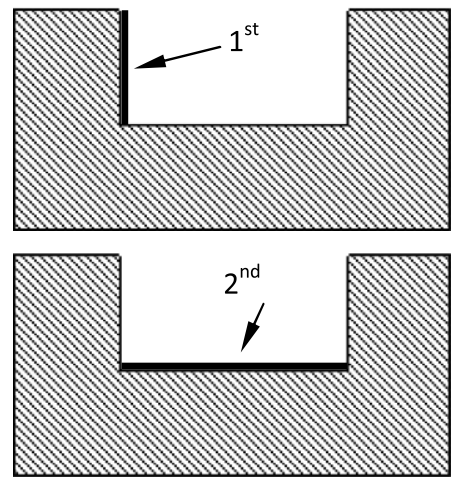

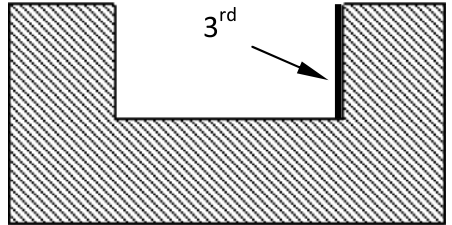

(a)

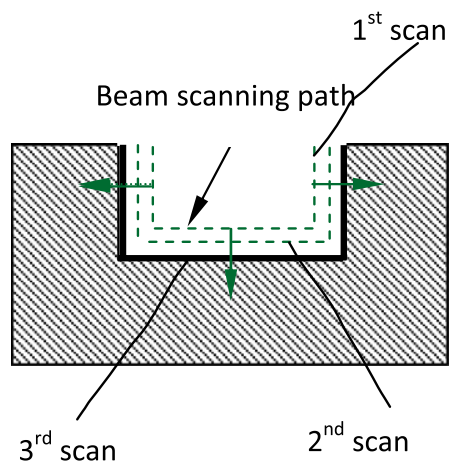

$(c)$

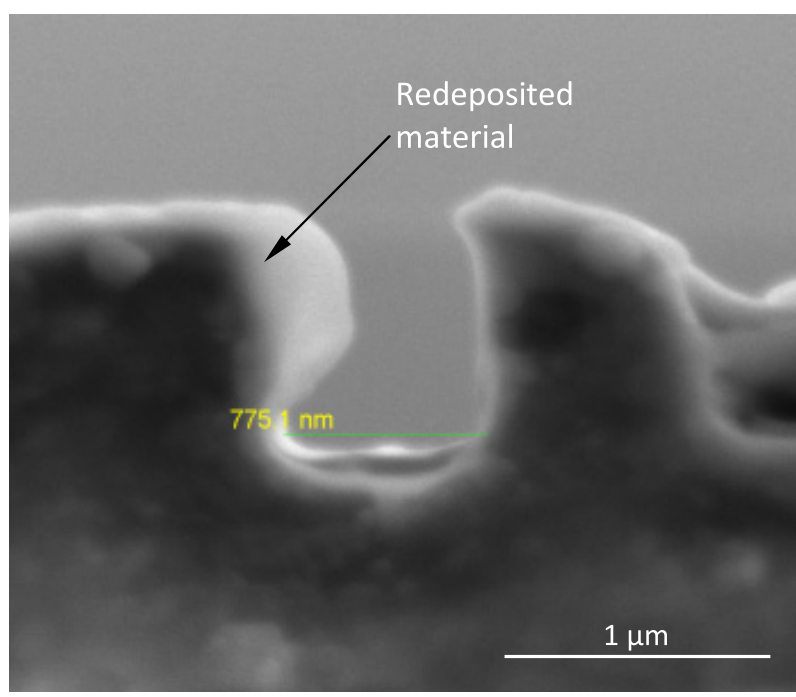

(b)

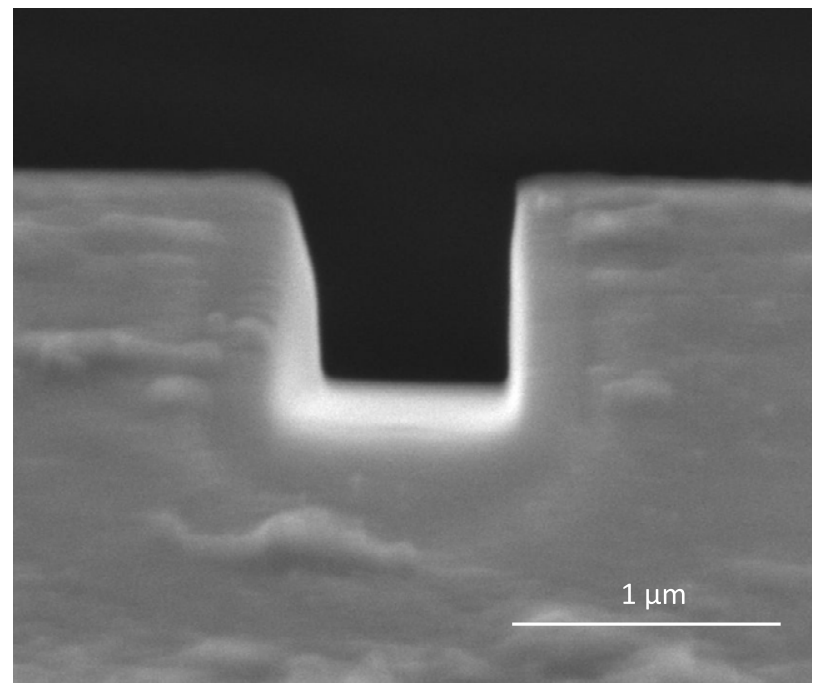

$(d)$

Figure 5. FIB polished nanostructures by applying different scanning strategies. $(a)$ and $(b)$ An 'edge-to-edge' polishing process and polishing result achieved; $(c)$ and $(d)$ A 'multi-edge' polishing process and polishing result achieved.

to view the moving front as a particular level set of a higher dimensional level set function $\phi$. The moving front $(x, y)$ is naturally embedded into this function. The velocity on the moving front $v_{\perp}$ can be obtained by solving the following equation:

$$
\left\{\begin{array}{l}
v_{\perp}(x, y, t)|\nabla \phi(x, y, t)|+\phi_{t}(x, y, t)=0 \\
\phi(x, y, t=0)=\Gamma
\end{array},\right.
$$

where $\Gamma$ is a customized initial state of the level set function. The moving front evolution can be deduced by monitoring certain level set ${ }^{5}$ of the level set function.

In this work, level set method has been applied in a surface topography model to simulate the contribution from the beam tail effect in ion polishing process. The moving front represents the cross-sectional profile of the diamond edge. The simulation imitated a beam of ions with a kinetic

\footnotetext{
5 In this work, the moving front is embedded in the zero level set.
}

energy of $30 \mathrm{keV}$ bombarded onto an abrupt diamond edge. In this simulation program, the ion current was set at $50 \mathrm{pA}$ which corresponds to a beam diameter of $19 \mathrm{~nm}$. In the surface topography evolution process, the time step and the grid size were set to $25 \mu \mathrm{s}$ and $1 \mathrm{~nm} \times 1 \mathrm{~nm}$, respectively. The crosssectional profile of the polished edge is directly embedded in the zero level set in the initial status. A comparison of the cross sections sputtered by the beam tail is shown in figure 6 . Figures $6(a)-(e)$ illustrates the evolution process of a beam of incident ions shoot along the $y$ direction. The centre of the beam is constrained at $x=0$. The cross-sectional view of the erosion on the abrupt edge caused by the beam tail is obtained by tracking the zero-level set of the level set function. The simulation results indicate that the beam tail effect is much more pronounced at a higher ion dose. As the incident ion dose increases, the sidewall of the edge became more abrupt. Meanwhile, the erosion area is also broadened. For a beam of focused ions with $19 \mathrm{~nm}$ diameter, only half of the beam 


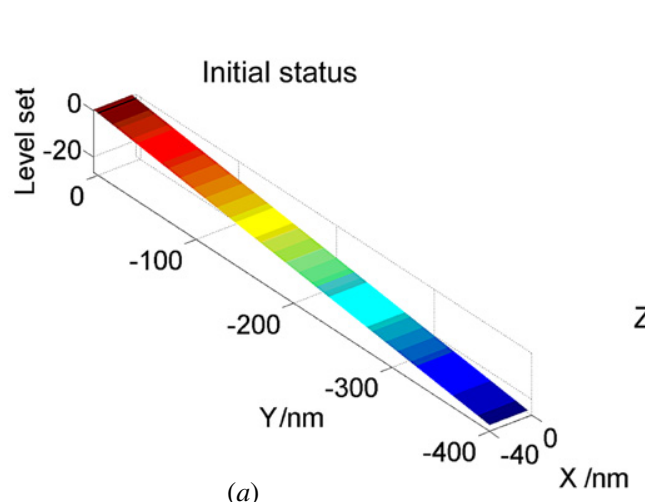

(a)

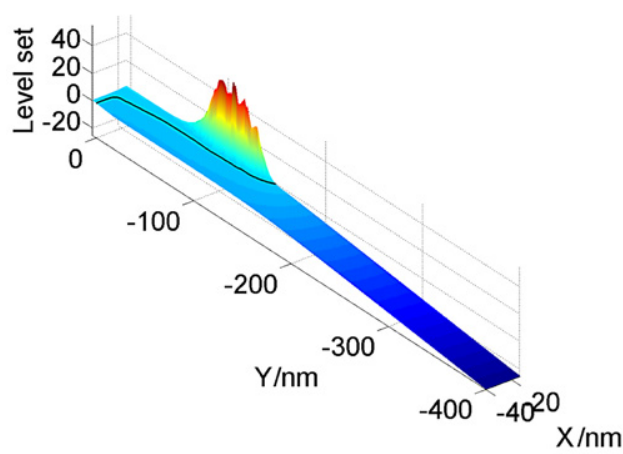

(c)

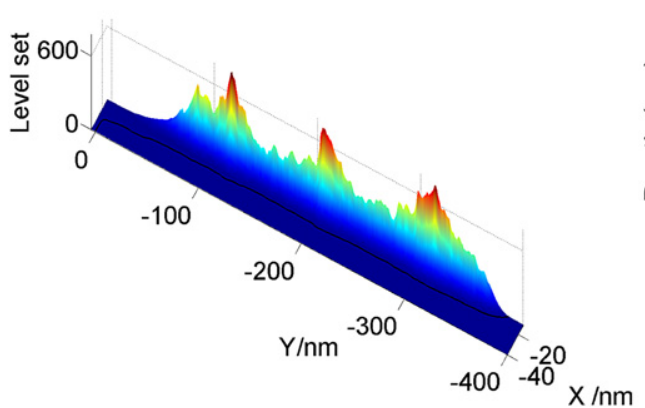

(e)

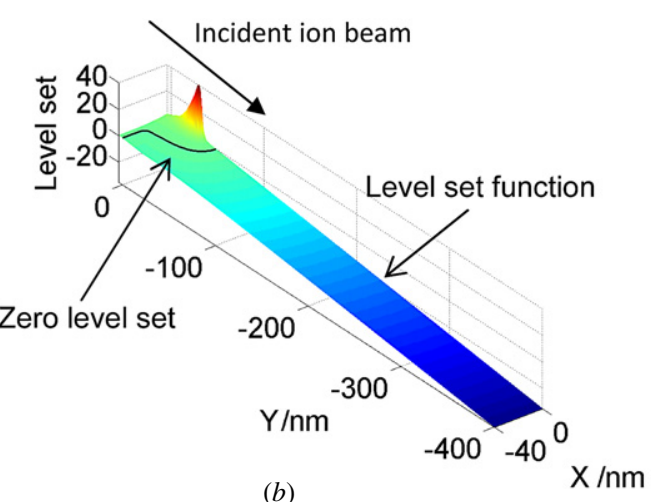

(b)

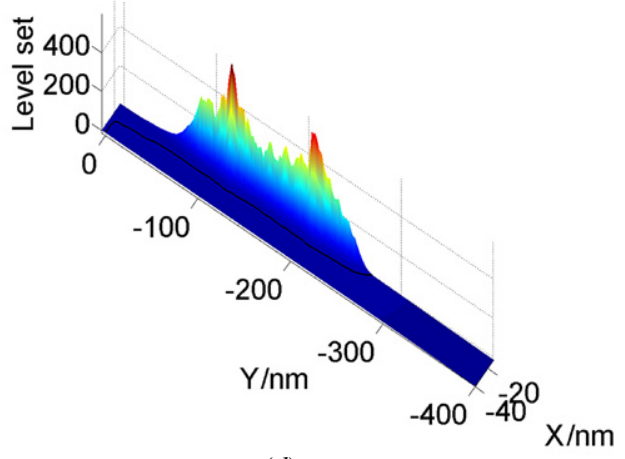

(d)

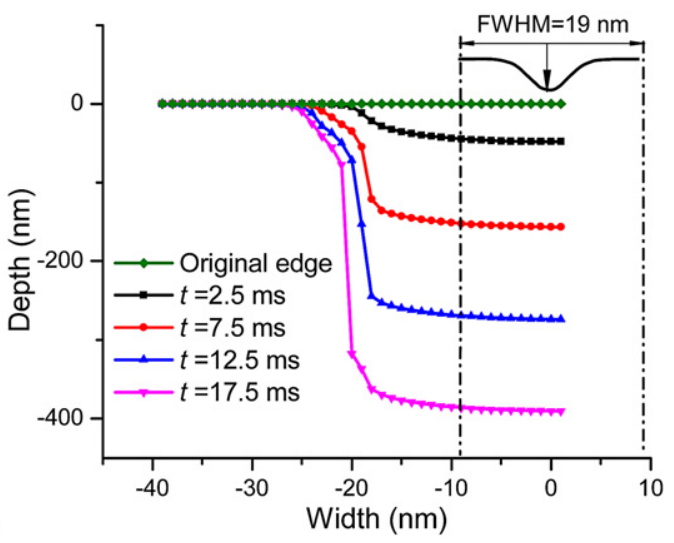

(f)

Figure 6. The level set function and the cross-sectional profile of the edge (the zero level set) when fabricated by a beam of ions with a diameter of $19 \mathrm{~nm}$ at different dwell time $t$. $(a) t=0 \mathrm{~ms},(b) t=2.5 \mathrm{~ms},(c) t=7.5 \mathrm{~ms},(d) t=12.5 \mathrm{~ms},(e) t=17.5 \mathrm{~ms}$ and $(f)$ comparison of the cross-sectional profiles at different dwell time.

$(9.5 \mathrm{~nm})$ can impact on the edge. However, the erosion can expand to $25 \mathrm{~nm}$ away from the edge. It can be estimated that for diamond substrate the erosion area caused by the beam tail is about three times of the beam radius. A positive offset along the normal direction of the tool's profile can be used to compensate this effect. According to the simulation result, a positive offset of $25 \mathrm{~nm}$ was applied in this experiment.

\subsection{Fabrication results}

For the sake of comparison, the original SCD tool is shown in figure $7(a)$. Figures $7(b)$ and $(c)$ show SEM images of a FIB fabricated nano-SCD tool under different magnifications and different views. An ion induced secondary electron image (taken from the clearance face) of the diamond tip with a magnification $20000 \times$ is shown in figure $7(d)$. Periodical chisel structures with finely finished edges are successfully obtained. The ripple-free sidewalls on the diamond tip are clearly observed in figure $7(c)$. The surface texture of the diamond tip was measured by a white light interferometer. A surface roughness $(\mathrm{Ra})$ of $1 \mathrm{~nm}$ is obtained on both clearance face and rake face.

Both the structure profile and the cutting edge radius of the diamond tip were measured by the SEM which is built inside the dual beam system. After the FIB sputtering process, the tool was flipped $180^{\circ}$ for the measurement. The SEM image is taken from the rake face where the sharp cutting edge is situated. The measurement results of the form accuracy are shown in 


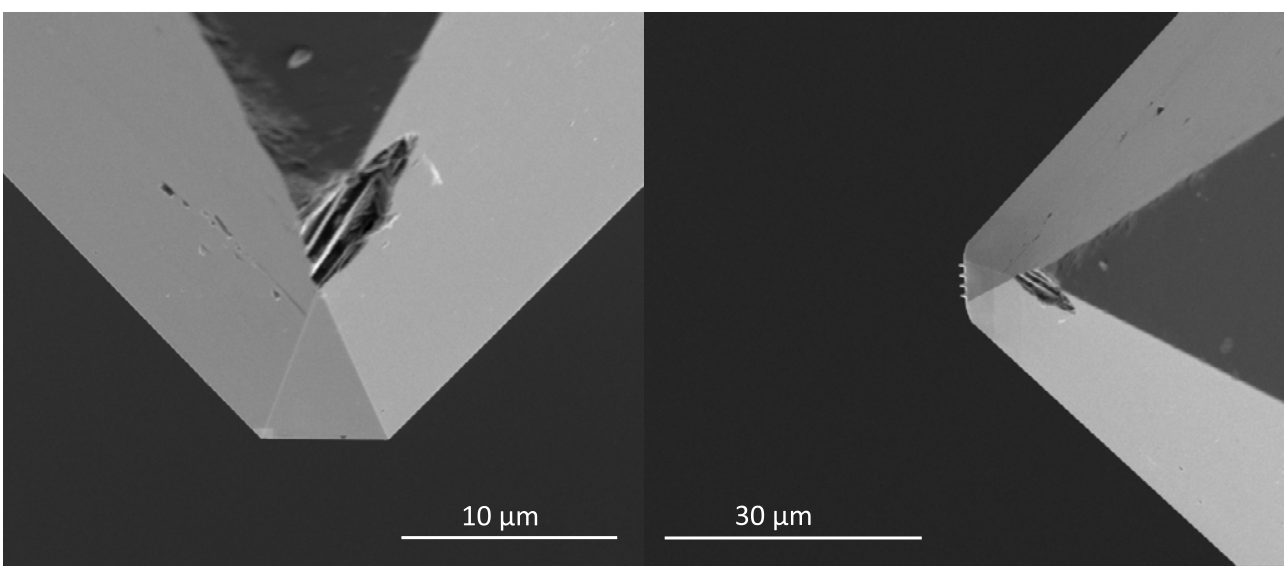

(a)

(b)

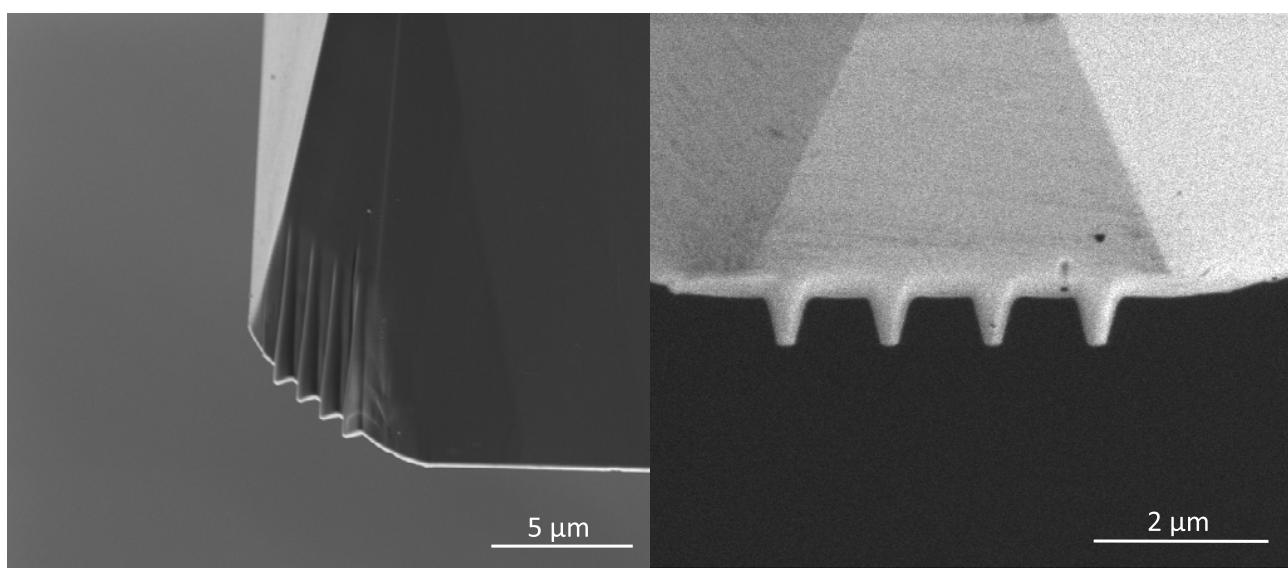

(c)

$(d)$

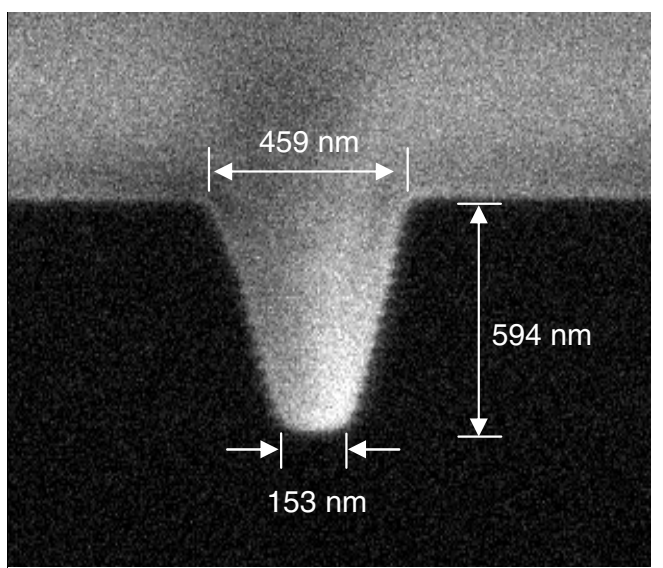

$(e)$

Figure 7. Nanoscale SCD tool fabricated by FIB machining. (a) Original SCD tool; $(b)$ a lower magnification view of the same tool trimmed by FIB; $(c)$ and $(d)$ lateral and top view of the nanoscale SCD tool and $(e)$ a close up view of the nano diamond tip.

Table 1. The relative error of the fabricated nanostructure on the diamond tip.

\begin{tabular}{lcll}
\hline Tip number & Tip width (\%) & Base width (\%) & Tip length $(\%)$ \\
\hline 1 & 1.9 & 1.9 & -1.0 \\
2 & 4.3 & 3.6 & -1.0 \\
3 & 3.5 & 3.6 & -1.0 \\
4 & -1.4 & 0.4 & -1.7 \\
\hline
\end{tabular}

table 1 . The relative error is controlled within $-1.7 \%-4.3 \%$. The maximum divergence on the tip is $-10.2 \mathrm{~nm}$. This value is very close to the ion beam resolution which is $7 \mathrm{~nm}$ at $30 \mathrm{kV}$ at beam coincident point ${ }^{6}$. The tool's cutting edge was cleaned using an ion current of $10 \mathrm{pA}$ from the clearance face. The measurement result shows that the tool cutting edge radius is achieved at a minimum value of less than $40 \mathrm{~nm}$.

6 The coincident point is the optimal working point of dual beam system that both the ion beam and the electron beam focus on at the same point. At the coincident point, the beam remains focused and almost does not shift when the stage is tilted or rotated in any direction. 


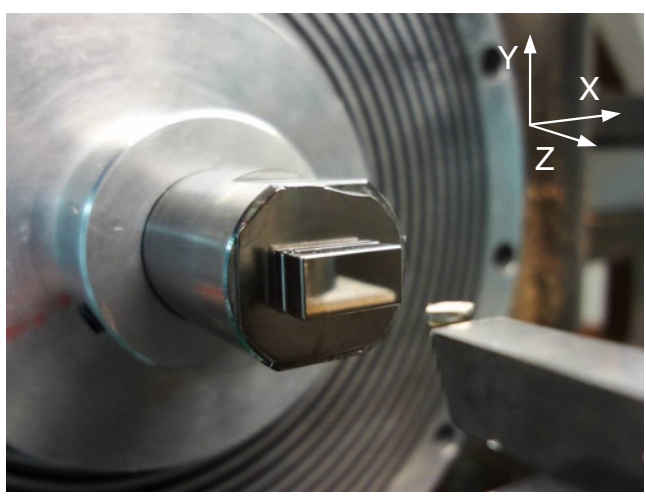

(a)

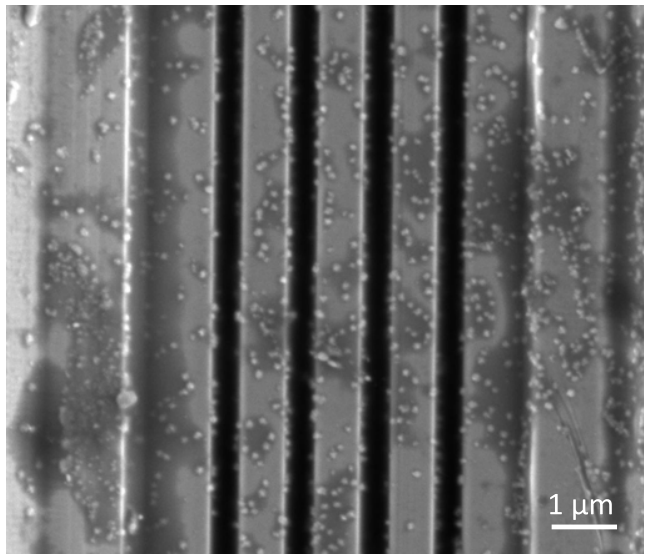

(c)

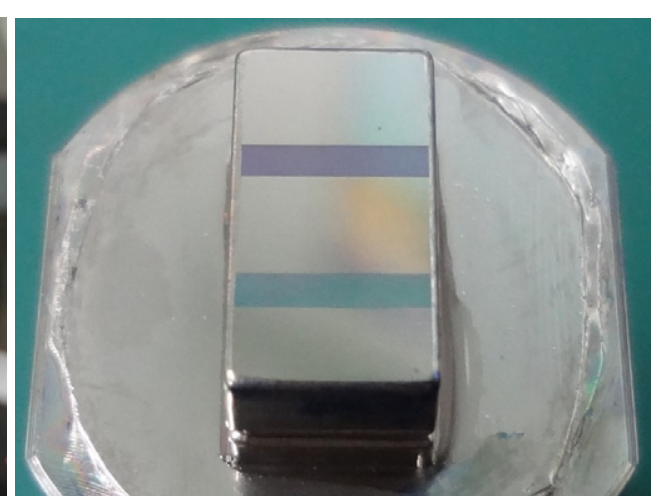

(b)

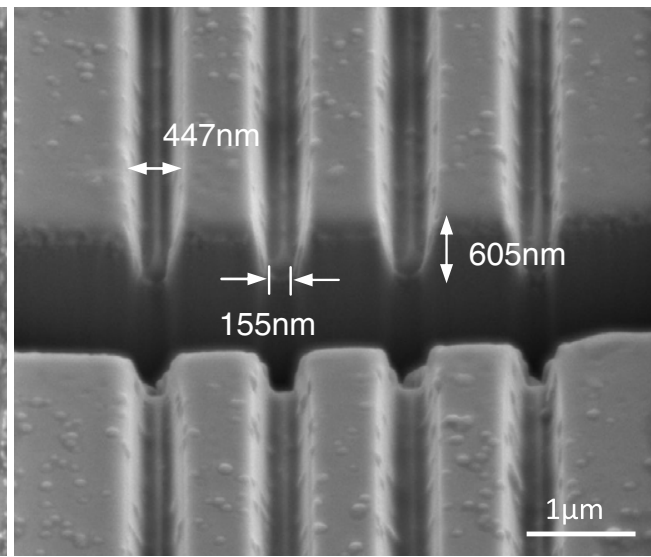

$(d)$

Figure 8. Nano-grating array turned by the nanoscale SCD diamond tool: $(a)$ experimental setup of the SPDT test; (b) nano-grating arrays were fabricated in two regions on the sample surface; $(c)$ the SEM image of the nano-grating array and $(d)$ cross-sectional view of the nano-grating array.

The cutting performance of the nanoscale SCD tool was tested on a Precitech Freeform 700 ultra precision diamond turning machine through machining nano-grating array on an electroless nickel substrate which was mounted on a vacuum chuck of the machine spindle. As shown in figure 8(a), the diamond tool was setup on the $Z$ axis and its tip was vertical to the machined surfaces. During the machining process, ' $C$ axis mode' was used so the spindle was locked into the position. The depth of the cut and cutting speed were chosen as $0.1 \mu \mathrm{m}$ and $1000 \mathrm{~mm} \mathrm{~min}^{-1}$, respectively. Cutting fluid (Mobil Velocity Oil No.10) was applied in the machining process. Through the $Y$-axis motion, nano-grating array was obtained on the nickel substrate (as shown in figure $8(b)$ ), where colourful interference fringes can be clearly observed. An SEM image of the nano-grating array with a magnification of 15000 is shown in figure $8(c)$. Figure $8(d)$ shows the cross-sectional view of the nano-grating array. The image was taken by the SEM with a relative angle of $38^{\circ}$ to the substrate surface. Nano-grating array with good form coherence was successfully obtained. The measurement indicates that the depth of the nano-gratings is $605 \mathrm{~nm}$, while the mouth width is $447 \mathrm{~nm}$ which is $13 \mathrm{~nm}$ less than the bottom width of the tool tip. This divergence is believed to be caused by an elastic recovery that occurred upon the surface of electroless nickel when the tool tip was released from the cutting track. Using such a nano-scale diamond tool in diamond turning process to fabricate nanostructures, the material removal rate (MRR) is

$$
\text { MRR }=4 S_{\mathrm{cs}} v_{c}=1.8 \times 10^{4} \mu \mathrm{m}^{3} \mathrm{~s}^{-1},
$$

where $S_{\mathrm{sc}}$ is the cross-section area of each nanostructure and $v_{c}$ is the cutting speed. The material removal rate is 30000 times higher than purely FIB machining with ion current of $1 \mathrm{nA}$.

\section{Conclusions}

A hybrid scale up approach has been successfully developed to fabricate nano-grating arrays over large area. This technique uses our deterministic fabrication method combined with a surface topography simulation method to overcome the addressed challenges in focused ion beam (FIB) nanofabrication process, thereby obtaining nano-scale structures with high accuracy on SCD tools. The surface roughness Ra is $1 \mathrm{~nm}$ while the cutting edge radius is controlled less than $40 \mathrm{~nm}$. In the fabrication process, it is found that grazing incidence $\left(>85^{\circ}\right)$ with low ion current is helpful in forming a ripple free diamond surface. The redeposition effect can be efficiently suppressed by the 'multi-edge' polishing method. In the FIB machining process, a beam of focused ions with $19 \mathrm{~nm}$ diameter can cause an erosion area expanding to $25 \mathrm{~nm}$ away from the edge. By using the developed fabrication technique, a form accuracy of $10 \mathrm{~nm}$ is achieved on the 
diamond tip. Periodic nano-gratings with a mouth width of $447 \mathrm{~nm}$, a bottom width of $155 \mathrm{~nm}$ and a depth of $605 \mathrm{~nm}$ were fabricated on an electroless nickel substrate by the developed single crystal diamond tool. The total machining area is $5 \times 2 \mathrm{~mm}^{2}$ which was completed within $2 \mathrm{~min}$

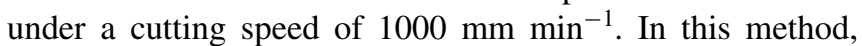
the material removal rate is 30000 times higher than purely FIB machining (with ion current of $1 \mathrm{nA}$ ). Moreover, there is a great potential to further boost the machining speed by simply adding more structures on the tool's tip. The experimental results show that it is very promising to apply this scale up approach for fabricating nanostructures accurately and cost effectively. Furthermore, the present technique also opens up the possibility of creating patterns on a precision drum, which can be further applied to imprint nanostructures over extreme large area by a roll-to-roll process.

\section{Acknowledgment}

The authors gratefully acknowledge the financial support from EPSRC's IMRC at Heriot-Watt University (Project no 113955) and Royal Society of Edinburgh (Project no 113666).

\section{References}

Adams D P, Vasile M J, Benavides G and Campbell A N 2001 Micromilling of metal alloys with focused ion beam-fabricated tools Precis. Eng. 25 107-13

Adams D P, Vasile M J and Krishnan A S M 2000 Microgrooving and microthreading tools for fabricating curvilinear Precis. Eng. 24 347-56

Akimov Y A and Koh W 2010 Resonant and nonresonant plasmonic nanoparticle enhancement for thin-film silicon solar cells Nanotechnology 21235201

Allen D, Shore P, Evans R, Fanara C, O'Brien W, Marson S and O'Neill W 2009 Ion beam, focused ion beam, and plasma discharge machining CIRP Ann.-Manuf. Technol. 58 647-62

Atwater H A and Polman A 2010 Plasmonics for improved photovoltaic devices Nature Mater. 9 205-13

Bradley R M and Harper J M E 1988 Theory of ripple topography induced by ion bombardment J. Vac. Sci. Technol. A $62390-95$

Cheung C F and Lee W 2003 Surface Generation in Ultra-Precision Diamond Turning: Modelling and Practices (New York: Wiley)

Chou S Y, Krauss P R and Renstrom P J 1995 Imprint of sub 25 nm vias and trenches in polymers Appl. Phys. Lett. 673114

Chou S Y, Krauss P R and Renstrom P J 1996 Nanoimprint lithography J. Vac. Sci. Technol. B 14 4129-33

Chou S Y, Krauss P R, Zhang W, Guo L and Zhuang L 1997 Sub-10 nm imprint lithography and applications J. Vac. Sci. Technol. B 15 2897-904

Clark A W and Cooper J M 2011 Nanogap ring antennae as plasmonically coupled SERRS substrates Small 7 119-25
Ding X, Lim G C, Cheng C K, Butler D L, Shaw K C, Liu K and Fong W S 2008 Fabrication of a micro-size diamond tool using a focused ion beam J. Micromech. Microeng. 18075017

Dubey A K and Yadava V 2008 Laser beam machining-a review Int. J. Mach. Tools Manuf. 48 609-28

Evans R W, Marson S and Allen D M 2006 A review of focused ion beam technology for the fabrication of ultra precision diamond cutting tools 6th Int. Conf. on Materials for Microelectronics and Nanoengineering (Cranfield, UK) pp 9-12

Krebs F C, Gevorgyan S A and Alstrup J 2009 A roll-to-roll process to flexible polymer solar cells: model studies, manufacture and operational stability studies J. Mater. Chem. $195442-51$

Liu Z, Steele J M, Srituravanich W, Pikus Y, Sun C and Zhang X 2005 Focusing surface plasmons with a plasmonic lens Nano Lett. 5 1726-9

Mokkapati S, Beck F, Polman A and Catchpole K 2009 Designing periodic arrays of metal nanoparticles for light-trapping applications in solar cells Appl. Phys. Lett. 95053115

Möller W and Eckstein W 1984 Tridyn-A TRIM simulation code including dynamic composition changes Nucl. Instrum. Methods Phys. Res. B 2 814-8

Möller W, Eckstein W and Biersack J P 1988 TRIDYN-binary collision simulation of atomic collisions and dynamic composition changes in solids Comput. Phys. Commun. $51355-68$

Nakamura T, Kanada Y and Nakai T 1994 Polycrystalline diamond cutting tool and method of manufacturing the same US Patent 5366522

Picard Y N, Adams D, Vasile M and Ritchey M 2003 Focused ion beam-shaped microtools for ultra-precision machining of cylindrical components Precis. Eng. 27 59-69

Sethian J 2000 Level Set Methods and Fast Marching Methods: Evolving Interfaces in Computational Geometry, Fluid Mechanics, Computer Vision, and Materials Science (Cambridge: Cambridge University Press)

Sun J, Luo X, Ritchie J, Chang W and Wang W 2011a An investigation of redeposition effect for deterministic fabrication of nanodots by focused ion beam Precis. Eng. 36 31-36

Sun J, Luo X, Ritchie J and Hrncir T $2011 \mathrm{~b}$ A predictive divergence compensation approach for the fabrication of three-dimensional microstructures using focused ion beam machining Proc. Inst. Mech. Eng. B 226 229-38

Tseng A A 2005 Recent developments in nanofabrication using focused ion beams Small 1 924-39

Vasile M J, Friedrich C R, Kikkeri B and Mcelhannon R 1996 Micrometer-scale machining: tool fabrication and initial results Precis. Eng. 19 180-6

Volkert C A and Minor A M 2007 Focused ion beam microscopy and micromachining MRS Bull. 32 389-9

Xu Z, Fang F, Zhang S, Zhang X, Hu X, Fu Y and Li L 2010 Fabrication of micro DOE using micro tools shaped with focused ion beam Opt. Express 18 8025-32

Yan Y, Hu Z, Zhao X, Sun T, Dong S and Li X 2010 Top-down nanomechanical machining of three-dimensional nanostructures by atomic force microscopy Small 6 724-8

Ziegler J F 2011 Element Database of TRIM 